in die Kompetenzen der jeweiligen Entwicklungsländer einzugreifen? Was sollen die Anspruchsgegner überhaupt tun? Ein so komplexes Geflecht kann nicht mit einem neuen Menschenrecht aufgelöst werden. Benedek ist dann auch skeptisch (S. $307 \mathrm{ff}$.) und will wenn überhaupt - die Entwicklungsländer selbst als Treuhänder der Entwicklungsansprüche ihrer Bürger auftreten lassen. Angesichts der bisherigen Praxis stellt sich dann allerdings die Frage, ob damit nicht die Erfüllbarkeit des Anspruchs endgültig vereitelt wird. Das gilt um so mehr, als jede Hilfe aus dem Norden, bevor sie bei den Menschen in Afrika ankommt, notwendig eine oder mehrere Qualitätsänderungen durchmacht: Ein Dollarbetrag schafft in keinem Dorf einen neuen Brunnen oder eine Meerwasserentsalzung. Hier sind Betroffene, vorhandene Administration und subsidiäre personelle Hilfe von außen erforderlich. Jedes Recht auf Entwicklung bleibt so notwendig eine leere Hülse: Menschen oder Gemeinwesen können sich entwickeln, aber nicht entwickelt werden. Entwicklungsvölkerrecht ist ein Prinzip jedes zukünftigen wie auch des geltenden Völkerrechts. Hierfür Instrumente zu entwickeln, sind Rechtsordnungen und Rechtswissenschaft aufgerufen. Das Recht auf Entwicklung gehört - als subjektives Recht von Menschen oder Staaten - nicht dazu.

Der vorliegende Band sucht zu neueren Grundrechtsproblemen zum Teil neue Antworten. Gerade die vergleichenden Abhandlungen stellen einen wesentlichen Fortschritt für die deutsche Rechtswissenschaft dar. Zeigt sie sich gerade in Grundrechtsfragen oft überaus introvertiert, so lassen sich hier einem Vergleich neue Perspektiven abgewinnen, die den Horizont zu erweitern vermögen. Und das war eine wesentliche Aufgabe der Tagung.

Christoph Gusy

\title{
Günther Rusch
}

Die verhinderte Mitsprache - Aspekte zur Sprachpolitik in Ghana, Togo und Obervolta Arbeiten aus dem Institut für Afrika-Kunde; Bd. 47, Hamburg 1984

Schon der Titel des Buches gibt Auskunft über die Einschätzung des Autors bezüglich des behandelten Themas, ist er doch Ausdruck dafür, daß die Bevölkerung - wie Rusch es formuliert - in Ghana, Togo und Obervolta bei den augenblicklichen sprachpolitischen Gegebenheiten "nur schwerlich die Kenntnisse und Informationen erhält, um den neuen Staat oder zumindest die regionale sozio-ökonomische Entwicklung bewußt mitzutragen" (p. 4). Was Rusch hier auf drei Länder bezieht, hat in gleicher oder ähnlicher Form Gültigkeit für die Mehrheit der schwarzafrikanischen Staaten.

Ursache des Problems ist die Tatsache, daß die Mehrzahl der afrikanischen Staaten eine starke linguistische Zersplitterung aufweist, daß also in jedem Land eine Vielzahl von Sprachen Verwendung findet. Für die drei untersuchten Länder können (nach Dalby) nicht weniger als 127 einheimische Sprachen und Dialekte aufgelistet werden. Daß da- 
durch innerstaatliche Kommunikationsprobleme entstehen, braucht nicht im Einzelnen erläutert zu werden. Verwiesen sei nur auf den Verwaltungs- und Schulbereich. Weitergehende Implikationen werden von Rusch dergestalt umschrieben, daß er "Sprachpolitik unabdingbar mit nationalen oder regionalen Entwicklungsstrategien verbunden" (p. 5) sieht.

Der eigentliche Kernpunkt des Problems ist jedoch der Umstand, daß die politisch relevanten Kräfte in den einzelnen betroffenen Staaten nicht willens oder in der Lage sind, sich auf eine oder mehrere der autochthonen Sprachen als Nationalsprache zu verständigen, und dieser/diesen im Gefolge durch entsprechende Maßnahmen ein entsprechendes Gewicht zu verleihen.

Nach der Darstellung der linguistischen Situation in Ghana, Togo und Obervolta (die Gründe für die Auswahl dieser drei Staaten liegen in geografischen, historischen und sozio-ökonomischen Gemeinsamkeiten. Außerdem verbietet sich eine isolierte Betrachtung eines Staates angesichts der zahlreichen grenzüberschreitenden Sprachen), unterzieht Rusch die Situation in den drei Ländern während der Kolonialzeit einer detaillierten Analyse. In allen drei Kolonialgebieten verfolgten die europäischen Mächte England, Frankreich und bis 1914 auch Deutschland einen vergleichbaren Ansatz. Es ging ihnen um die Stabilisierung und Sicherung ihrer Macht. Dabei wurde den autochthonen Sprachen eine unterschiedliche Rolle zugewiesen. Während Großbritannien im Rahmen der indirect rule` zwar Englisch zur Amtssprache erhob, kam es andererseits dazu, daß "in zunehmendem Maße die Verwendung von afrikanischen Sprachen als Schulsprachen unterstützt" (p. 46) wurde. Das gilt insbesondere für Asante, Twi und Fante, die noch heute zu den linguistisch am besten erforschten Sprachen Afrikas zählen. Ähnliches läßt sich auch über das Ewe sagen. Diese in Togo und Ghana verbreitete Sprache profitierte bis 1914 vor allem durch die Förderung, die ihr - zweckgebunden - durch evangelische Missionare zuteil wurde. Anders stellte sich die Situation in den französischen Kolonialterritorien dar. Dort wurde den einheimischen Sprachen wenig Gewicht beigemessen, Französisch zum alleinigen Maßstab erhoben. Man mag diese Politik der Kolonialmächte kritisieren, unbestreitbare Tatsache ist jedoch, daß - wie Rusch auch ausführt sich die europäischen Kolonisatoren durchaus im Einklang mit den Vorstellungen "traditioneller Autoritäten" sowie "städtisch orientierter Nationalisten" befanden, befürchteten diese doch "eine Infragestellung ihrer Positionen, wenn die Bevölkerung mündlich und schriftlich von ihrer eigenen Sprache zum Ausdruck ihrer Unzufriedenheit Gebrauch machte." (p. 82)

Einen überraschenden Fortgang nimmt dieses Thema jedoch, wenn man das Ende der Kolonialära betrachtet und sich der Phase nach der Unabhängigkeit zuwendet. Auch dabei stehen Ghana (1957), Togo (1960) und Obervolta (1960) exemplarisch für das ganze subsaharische Afrika. Denn nimmt man einige Staaten aus (z. B. Tanzania, Kenya und Somalia), so stellt man fest, daß dieses Thema in den zweieinhalb Jahrzehnten Unabhängigkeit wenig an Virulenz verloren hat. Eigentlich hätte man eine Hinwendung in den einzelnen, unabhängig gewordenen Staaten zu einheimischen Sprachen vermuten kön- 
nen, doch läßt man die 25 Jahre Revue passieren, so wüberrascht der niedrige Stellenwert der afrikanischen Sprachen in der Diskussion um Unabhängigkeit und Souveränität“. (p. 80)

Zwar sieht Rusch die "augenfällige Vernachlässigung", die "unreflektierte Úbernahme metropolitaner Sprachpolitik“ zu Beginn der siebziger Jahre schwinden, doch nach Erlangung der Unabhängigkeit kam es zunächst einmal zu einer Verstärkung von Englisch bzw. Französisch in Unterricht und Verwaltung, was automatisch eine Minderung des Stellenwerts der lokalen Sprachen bewirkte. Damit wurden jedoch Tatbestände geschaffen, die die Folgezeit prägten.

Einheimische Sprachen konnten bei der Bevölkerung kein Interesse erwecken, war doch für jedermann offensichtlich, daß der Zugang zu relevanten Positionen im Staatsdienst und in der Wirtschaft nur über die Kenntnis europäischer Sprachen lief. So kann es auch nicht verwundern, daß grundsätzlich mehr Interesse besteht, im Rahmen von Alphabetisierungskampagnen oder in der Schule Englisch bzw. Französisch zu erlernen als die eigenen, nur lokal zu verwendenden Sprachen. Hinzu tritt die immer noch weitverbreitete Ansicht, die technischen Fortschritt und Zivilisation ausschließlich mit europäischen Sprachen assoziiert. Fehlende Aufklärung in der Bevölkerung wird im allgemeinen als Ursache für dieses mangelnde Eintreten für die eigenen Sprachen verantwortlich gemacht. Uberdies werden jede Menge technische und organisatorische Gründe - je nach Standpunkt - zur Erklärung oder Entschuldigung für die augenblickliche, unbefriedigende Situation herangezogen: fehlende linguistische Kenntnisse über einzelne Sprachen; Orthographieprobleme; der Mangel an qualifizierten Lehrern und letztlich stehen - im Gegensatz zu Englisch und Französisch - nicht genügend publizierte Materialien für alle Fächer und Unterrichtsstufen zur Verfügung.

Doch handelt es sich bei all diesen Aufzählungen nicht um Beschönigungen seitens der Verantwortlichen, liegt die Ursache der innerstaatlichen Sprachproblematik in Afrika nicht auf einer anderen Ebene? Rusch bezieht auch diesen Aspekt mit ein, wenn er z. B. schreibt, daß Grundsatzentscheidungen unterblieben oder "Entscheidungen mehr als Konfliktthema und weniger als Mobilisierungsmöglichkeit" (p. 98) angesehen wurden. Doch gerade hier liegt die Schwachstelle in Rusch Darstellung, denn in additiver Form werden einzelne Verhinderungselemente einer sinnvollen Sprachpolitik aufgelistet. Was fehlt, ist eine konsistente Bewertung. Verhinderte Mitsprache, so lautet der Titel. Doch wer verhindert? Sind es die Umstände, Strukturen oder Personen? Sicherlich gibt es Zusammenhänge, doch leider stellt Rusch dieses Verflechtungsnetz nicht in der Stringenz dar wie er mit Vehemenz die Sprachpolitik der ehemaligen Kolonialmächte verurteilt. Und so weiß man trotz aller Einzelinformationen nicht, was letztlich die entscheidenden Hindernisse sind, eine den nationalen Interessen entsprechende Sprachpolitik in die Tat umzusetzen. Ursache hierfür ist der Umstand, daß Rusch sich auf die Beschreibung von Oberflächenphänomenen beschränkt. Das ist bei der gegebenen Quellenlage nicht verwunderlich. Und so vermerkt der Autor zu Beginn des Buches: "Notgedrungen wird deshalb die Beschreibung der Sprachpolitik nicht so sehr Auswertung und Analyse von 
sprachpolitischen Maßnahmen sein können, sondern eher versuchen, Sprachpolitik als Phänomen der Staatswerdung zu schildern und Gemeinsamkeiten zwischen Kolonialzeit und der Gegenwart aufzuzeigen" (p. 8).

Ansätze, Sprache als Vehikel zur Staatswerdung zu nutzen, gab es wie schon zuvor angemerkt ab etwa 1970. Unter dem Schlagwort der kulturellen Identität kam es auch zu einer Rückbesinnung auf die autochthonen Sprachen. Doch die bisherigen Ergebnisse solcher Kampagnen deuten eher darauf hin, daß es sich um Lippenbekenntnisse handelt.

Zuweilen kommt es zu Programmen, die die Verankerung der lokalen Sprachen in der Bevölkerung zum Ziel haben. Doch selbst dann sind Zweifel bezüglich der Zielsetzung begründet. Rusch verweist in diesem Zusammenhang auf die unterschiedlichen Interessenstrukturen (Staat versus Bevölkerung), die z. B. im Rahmen der Alphabetisierungskampagne in Togo deutlich zutage traten. In dem jüngst erschienenen Buch "Bauern und Staat" macht G. Elwert entsprechend entmutigende Angaben zu seinen Erfahrungen bezüglich einer gesellschaftsformenden und entwicklungstragenden Möglichkeit von Alphabetisierung und Förderung autochthoner Sprachen.

Das alles wirft die Frage auf, ob die Mutmaßung Ruschs, es bestehe in den afrikanischen Staaten ein Interesse an "kultureller wie sozialer Selbstentfaltung" bezogen auf Sprache nicht doch ein realitätsfremder Ansatz ist bzw. zumindest eine entsprechende Verkürzung impliziert. Denn diese Sichtweise unterstellt ein einheitlich geartetes Entwicklungsinteresse in den einzelnen afrikanischen Ländern. Verschiedene Analysen haben jedoch gezeigt, daß das nicht gegeben ist. Und auch das besprochene Buch läßt eigentlich nur das traurige Fazit zu, daß Sprachpolitik heute - ebenso wie während der Kolonialzeit zweckgebunden eingesetzt wird. Bedenklicherweise jedoch nicht in Blickrichtung auf eine umfassende Entwicklung von Staat und Gesellschaft, sondern wie ehedem im Sinne von Herrschaftsstabilisierung und -sicherung.

Thomas Labahn

\section{Susanne Weigelin-Schwiedrzik}

\section{Parteigeschichtsschreibung in der VR China, Typen, Methoden und Funktionen}

Veröffentlichungen des Ostasien-Instituts der Ruhr-Universität Bochum, Band 29, Wiesbaden: Otto Harrassowitz, 1984, pp xx, 248 (broschiert), DM 44,-

This doctoral dissertation is not, as the author herself emphasises, about the history proper of the Communist Party of China (CCP), but about the way in which the Party's past has been discussed and publicised in the People's Republic of China (PRC).

The book describes the types and media of historiography and examines the different groups of persons active on several levels in projecting an image of the CCP's history. For this purpose Ms Weigelin-Schwiedrzik has drawn on numerous publications - some 